\title{
Golimumab shows promise in treatment of active ulcerative colitis
}

Golimumab-a human monoclonal anti-TNF antibody-is effective for the treatment of patients with moderate-tosevere ulcerative colitis, according to new findings published in the journal Gastroenterology.

William Sandborn and colleagues integrated data from a phase II dose-finding trial and a phase III doseconfirmation trial to assess the efficacy of golimumab in 1,064 patients with ulcerative colitis. "The adaptive seamless [phase II/III] clinical trial design [is] an alternative concept to the traditional drug development programmes of sequential, independent clinical trials," say the authors of this study.

Patients with ulcerative colitis were randomly assigned to receive subcutaneous golimumab induction therapy at doses of $100 \mathrm{mg}$ then $50 \mathrm{mg}$ (phase II trial only), $200 \mathrm{mg}$ then $100 \mathrm{mg}$, or $400 \mathrm{mg}$ then $200 \mathrm{mg}, 2$ weeks apart. All patients included in the trials were naive to treatment with anti-TNF agents, and had moderate-to-severe disease activity

\section{The induction therapy was clinically effective ... however, we need to see the long-term maintanance data... 77}

(defined as a Mayo score of 6-12) despite conventional treatment.

Data from the phase II trial indicated that higher doses of serum golimumab (200 mg then $100 \mathrm{mg}$ and $400 \mathrm{mg}$ then $200 \mathrm{mg}$ ) led to clinical response and remission in more patients than a lower dose (100 mg then $50 \mathrm{mg}$ ), with no increase in adverse events.

In the phase III trial, the clinical response rate at 6 weeks after treatment (the primary end point) was significantly higher in patients given golimumab at doses of $200 \mathrm{mg}$ then $100 \mathrm{mg}(51.8 \%)$ or $400 \mathrm{mg}$ then $200 \mathrm{mg}(55.0 \%)$ than those given placebo (29.7\%; $P<0.001)$. All secondary end points, including rates of clinical remission and mucosal healing and IBD Questionnaire scores, were markedly increased in patients who received golimumab.
The rates of serious adverse events and serious infections were substantially lower in patients receiving golimumab than in those receiving placebo (3.0\% versus $6.1 \%$ and $0.5 \%$ versus $1.8 \%$, respectively).

"This study confirms that TNF is a key cytokine in the pathogenesis of ulcerative colitis and that its blockade with a monoclonal antibody can lead to treatment success," states Silvio Danese, an expert in the field who was not involved in this study.

"The induction therapy was clinically effective, with significant rates of mucosal healing and improvement in quality of life; however, we need to see the long-term maintanance data before we can make a full judgement," Danese adds.

Katherine Smith

\footnotetext{
Original article Sandborn, W. J. et al. Subcutaneous golimumab induces clinical response and remission in patients with moderate-to-severe ulcerative colitis. Gastroenterology doi:10.1053/j.gastro.2013.05.048
} 